\title{
Review \\ The protein C pathway: implications for the design of the RESPOND study
}

\author{
Burkhard Vangerow ${ }^{1}$, Andrew F Shorr ${ }^{2}$, Duncan Wyncoll ${ }^{3}$, Jonathan Janes ${ }^{4}$, David R Nelson ${ }^{5}$ \\ and Konrad Reinhart ${ }^{6}$
}

\author{
${ }^{1}$ Eli Lilly Critical Care Europe, Air Center, 1214 Vernier / Geneva, Switzerland \\ 2Walter Reed Army Medical Center, Pulmonary Medicine, 6900 Georgia Ave NW, Washington, DC 20307, USA \\ ${ }^{3}$ Guy's and St. Thomas' Foundation Trust, Adult Intensive Care Unit, Lambeth Palace Road, London SE1 7EH, UK \\ 4 Eli Lilly, Erl Wood Manor, Windlesham, Surrey GU20 6PH, UK \\ 5Lilly Research Laboratories, Eli Lilly and Company, Indianapolis, Indiana 46285, USA \\ ${ }^{6}$ Friedrich Schiller University, Clinic for Anaesthesiology and Intensive Care Medicine, Erlanger Allee 101, 07747 Jena, Germany
}

Corresponding author: Burkhard Vangerow, vangerow_burkhard@lilly.com

Published: 19 December 2007

This article is online at http://ccforum.com/content/11/S5/S4

(C) 2007 BioMed Central Ltd
Critical Care 2007, 11(Suppl 5):S4 (doi:10.1186/cc6155)

the systemic circulation initiate a cascade of events that results in widespread inflammation, endothelial injury and microvascular thrombosis $[2,3]$. Endogenous protein $\mathrm{C}$, a vitamin $\mathrm{K}$ dependent glycoprotein that is synthesized by the liver, plays an important role in the maintenance of haemostasis and modulation of inflammation under normal conditions [4,5]. Protein $\mathrm{C}$ circulates in the blood in zymogen form (inactivated form) at a concentration of approximately $4 \mu \mathrm{g} / \mathrm{ml}$. Currently, various methods, reagents and equipment exist to measure protein $\mathrm{C}$ in the laboratory or even at the bedside in the intensive care unit (ICU). In general, these methods can be subdivided into those that are based on measuring the functional activity of the protein (\%) and antigenic methods, which measure the amount of protein available $(\mu \mathrm{g} / \mathrm{ml})$.

Protein C is converted to activated protein C (APC) by thrombin-thrombomodulin complexes. APC inactivates coagulation factors $\mathrm{Va}$ and VIlla, thereby inhibiting further thrombin generation. Moreover, by inhibiting plasminogen activator inhibitor-1, APC is indirectly profibrinolytic. In addition to its role as an anticoagulant, a number of antiinflammatory effects and anti-apoptotic properties have been described [6]. Recent research indicates that inhibition of neutrophil chemotaxis and a role in maintaining endothelial integrity are other important effects of APC. Direct cytoprotective effects of APC have been reported to be mediated by intracellular signalling, initiated by binding of APC to its receptor (the endothelial protein $C$ receptor), which appears to be mediated by interaction with an adjacent protease-activated receptor or by indirect activation of the sphingosine 1-phosphate pathway $[7,8]$. 
Protein C is rapidly depleted in severe sepsis because of consumption, degradation by elastase and a reduced synthesis by the liver. With the reduction in protein $\mathrm{C}$ zymogen, the hypercoagulable condition and hyper-inflammatory response in sepsis is not counterbalanced, which leads to organ dysfunction and death in approximately $20 \%$ to $40 \%$ of cases.

Drotrecogin alfa (activated; DrotAA) is a recombinant version of endogenous human APC. The PROWESS (Recombinant Human Activated Protein C Worldwide Evaluation in Severe Sepsis) trial established that DrotAA (Xigris ${ }^{\circledR}$, Eli Lilly and Company, Indianapolis, IN, USA) is an effective treatment for adult patients with severe sepsis when it is infused at $24 \mu \mathrm{g} / \mathrm{kg}$ per hour for 96 hours [9], which is the currently licensed regimen. Thus far, the compound has been evaluated in more than 9,000 patients enrolled in clinical trials. Studies have considered the following issues: safety (ENHANCE [Extended Evaluation of Recombinant Human Activated Protein C, drotrecogin alfa (activated)] study [10]), use in patients who are less severely ill than the licensed indication (ADDRESS [Administration of Drotrecogin Alfa (Activated) in Early Stage Severe Sepsis] study [11]), paediatric use (RESOLVE [Researching severe Sepsis and Organ dysfunction in children: a global perspective] study [12]), interaction with prophylactic heparin (XPRESS [Xigris and Prophylactic Heparin Evaluation in Severe Sepsis] study [13]), and prolonged administration (EXTEND [Efficacy and Safety of Extended Drotrecogin Alfa (Activated) Therapy in Patients With Persistent Requirement for Vasopressor Support After 96 Hour Infusion With Commercial Drotrecogin Alfa (Activated)] [14]).

No biomarker has yet been established that can guide the clinical use of DrotAA. The identification of severe sepsis patients most likely to benefit from this drug is based mainly on clinical parameters. The phase II study RESPOND (Research Evaluating Serial Protein $\mathrm{C}$ levels in severe sepsis patients ON Drotrecogin alfa [activated]) [15] aims to evaluate the use of endogenous protein $C$ level as a biomarker to guide treatment with DrotAA.

\section{Protein C as a biomarker}

Abnormalities in coagulation parameters are commonly observed in patients with severe sepsis and have been associated with higher mortality. These include reductions of the major natural anticoagulants antithrombin III, tissue factor pathway inhibitor and protein C. Studies have shown that more than $80 \%$ of patients with severe sepsis had a baseline protein $C$ level below the lower limit of normal [9,16-18]. Plasma protein $\mathrm{C}$ levels decrease early in patients who develop severe sepsis, often before clinical symptoms appear, and these levels remain low, gradually rising in patients who recover and survive [18-21].

Numerous reports have examined the predictive value of plasma protein $\mathrm{C}$ levels in sepsis. These reports confirm the association between depressed protein C levels and the increased likelihood of negative outcomes in sepsis, including time on a ventilator, time in the ICU, development of shock and increased mortality [17,19,22-33]. Protein C levels less than approximately half the lower limit of normal are associated with a much greater risk for death $[19,29]$. In a recent multivariable analysis with ICU mortality as the dependent variable [32], a minimum protein $\mathrm{C}$ concentration of less than $45 \%$ was identified as an independent risk factor in surgical ICU patients.

Data from previous studies of DrotAA also show a strong association between very low protein $C$ levels and mortality in patients with severe sepsis. In the placebo arm of PROWESS [9] no difference in mortality was identified between patients with normal protein C levels $(>80 \%$ functional activity in the assay used) at baseline and those with moderate protein $\mathrm{C}$ deficiency ( $41 \%$ to $80 \%$ activity). In the former group, mortality was $5.7 \%$ at day 4 and $26.7 \%$ at day 28 , and in the latter group the corresponding percentages were $5.5 \%$ and $24.9 \%$, respectively. However, patients who presented with severe protein $\mathrm{C}$ deficiency (activity levels half the lower limit of normal or less $[\leq 40 \%]$ ) had a markedly higher mortality both at day 4 (20\%) and at day 28 (41.8\%).

Even though severe protein $\mathrm{C}$ deficiency at baseline clearly indicates a much greater risk for death, it is of note that there is an additional approximate $20 \%$ mortality between days 4 and 28 in all three groups, independent of baseline protein $\mathrm{C}$ levels. Based on this observation, additional analyses were performed to investigate whether sequential measurements of protein $\mathrm{C}$ levels would add further prognostic value. In PROWESS, protein $C$ levels were measured daily on study days 1 to 7, 14 and 28. Figure 1 shows sequential protein $C$ measurements in PROWESS placebo patients grouped by duration of survival [34]. In addition to the observation noted above that very low protein $\mathrm{C}$ levels predict early death, it was also demonstrated that survival beyond day 28 or hospital discharge was associated with an early return of endogenous protein $\mathrm{C}$ to above the lower limit of normal. Inability to increase endogenous protein $\mathrm{C}$ level to the normal range was indicative of poor outcome.

\section{Effect of drotrecogin alfa (activated) administration}

It is important to note that the activity assay utilized to measure protein $\mathrm{C}$ (the inactive zymogen) in the above studies is not subject to significant interference by therapeutic DrotAA (specifically, the assay reflects native endogenous protein $\mathrm{C}$ and not infusion of APC).

In PROWESS [9] the observed relative risk reduction in patients with protein $\mathrm{C}$ deficiency at baseline $(\leq 80 \%)$ was $20 \%$, whereas that in patients with normal protein $C$ levels at baseline (>80\%) was $42 \%$. There was no statistically significant treatment-by-subgroup interaction observed based on 
Figure 1

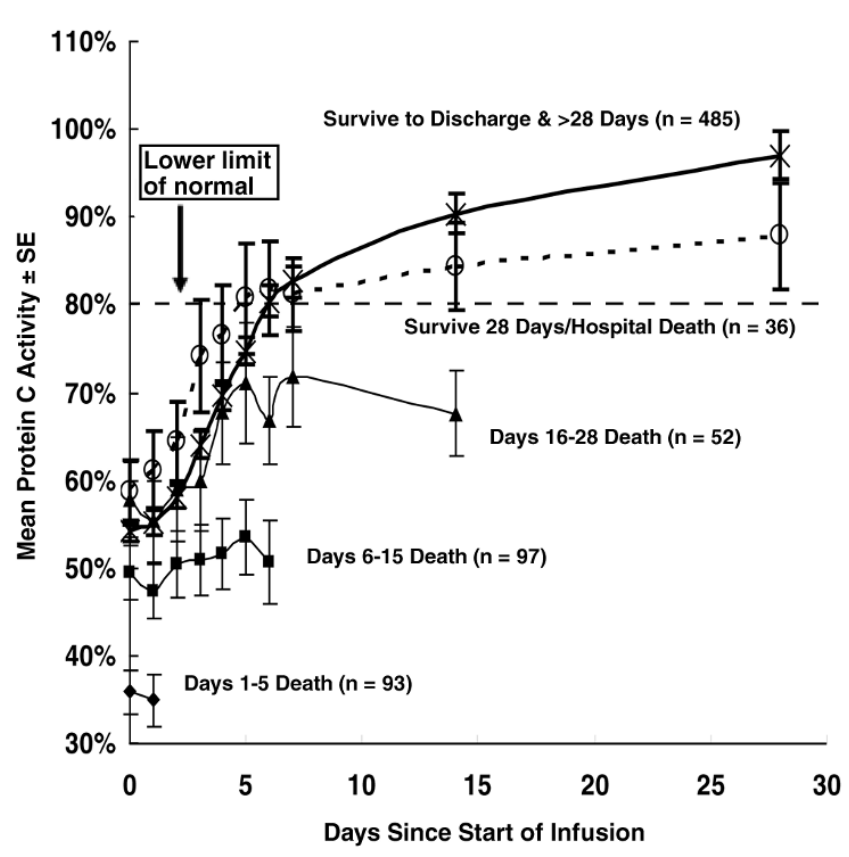

Mean protein $\mathrm{C}$ activity levels over time for PROWESS placebo patients classified according to timing of death [34]. Shown are means and standard errors (SE) of protein C levels based on time of death of PROWESS [9] drotrecogin alfa (activated) patients. Raw values with no imputation are included. PROWESS, Recombinant Human Activated Protein C Worldwide Evaluation in Severe Sepsis.

subgroups defined by baseline protein $\mathrm{C}$ deficiency status $(P=0.3338)$. This suggested that there was a treatment benefit associated with DrotAA administration in severe sepsis, irrespective of baseline protein $\mathrm{C}$ level. In an attempt to gain a better understanding of these findings, the changes in protein $\mathrm{C}$ levels from baseline to the end of study drug infusion were analyzed.

Patients were classified as to whether they had normal protein $\mathrm{C}$ levels at baseline or had a deficiency in protein $\mathrm{C}$, and whether they achieved normal protein $\mathrm{C}$ any time during infusion or had below normal levels at any time during infusion (Figure 2). In the group that was deficient in protein $\mathrm{C}$ at baseline, DrotAA patients were significantly more likely to improve to normal levels during infusion $\left(P=0.0001\right.$, by $\chi^{2}$ test); also, there was a trend $\left(P=0.14\right.$, by $\chi^{2}$ test) for more placebo patients with normal protein $C$ levels at baseline than DrotAA patients to exhibit below normal levels. For patients with below normal baseline protein $\mathrm{C}$ that remained below normal during infusion, there was no significant difference in 28-day mortality rates $\left(P=0.24\right.$, by $\chi^{2}$ test) between treatment groups. Similarly, for patients with normal baseline protein $\mathrm{C}$ that remained normal during infusion, there was no significant difference in 28-day mortality rates $(P=0.59$, by $\chi^{2}$ test) between treatment groups. Among those who improved from below normal protein $\mathrm{C}$ levels to above normal protein $C$ levels, DrotAA patients tended to have a lower mortality $\left(P=0.07\right.$, by $\chi^{2}$ test). DrotAA patients also had lower mortality among those who had above normal levels that fell below normal during infusion $(P=0.054)$. These findings have the following implications. First, the DrotAA treatment effect may be reflected, at least in part, by the increase in numbers of patients presenting with normal protein $\mathrm{C}$ levels at the end of infusion, who have generally low mortality. Second, DrotAA benefits patients with normal protein $\mathrm{C}$ levels at baseline because it reduces their likelihood of becoming deficient or may help to restore levels to normal by the end of infusion. Third, the benefit of DrotAA treatment is largely confined to patients who are, or who will become, protein $\mathrm{C}$ deficient.

Data from PROWESS and ENHANCE demonstrated that higher end-of-infusion protein $\mathrm{C}$ levels are associated with lower mortality (Figure 3). PROWESS also demonstrated that, compared with placebo patients, fewer DrotAA patients had severe protein $C$ deficiency at the end of the infusion (166 patients [19.5\%] versus 217 patients [25.8\%]), and a greater number of DrotAA patients had normal protein $\mathrm{C}$ levels at the end of the infusion (290 patients [34.1\%] versus 211 patients [25.1\%], $P<0.001)$. These findings were observed even though the DrotAA group had a greater number of patients with severe protein $C$ deficiency at baseline than did the placebo group (330 patients [38.8\%] versus 285 patients [33.9\%]). The protein $\mathrm{C}$ levels in DrotAA patients also rose more rapidly than did those in placebo patients (Figure 4) and were significantly higher on each day of the infusion period. There are a number of potential explanations for the observed effect of DrotAA administration on endogenous protein $C$ levels. It could be due to decreased consumption or degradation of endogenous protein $\mathrm{C}$ as the consumptive coagulopathy is inhibited; however, this may not be very relevant because the levels of other coagulation factors such as antithrombin III or protein $\mathrm{S}$ are not specifically increased with DrotAA treatment. Alternatively, there might be a direct effect of protein $C$ on hepatic synthesis.

Although most patients in PROWESS and ENHANCE had higher protein $\mathrm{C}$ levels at the end of the infusion, there remained a substantial number of patients who were protein $\mathrm{C}$ deficient despite treatment with DrotAA. Figure 5 shows the results of sequential protein $C$ measurements in patients treated with DrotAA. Analysis of protein $C$ levels in patients who died after completing the DrotAA infusion (during study days 6 to 15 ) revealed that protein $C$ levels gradually increased during the infusion, but mean protein $\mathrm{C}$ levels at the end of the infusion were less than three-quarters of the lower limit of normal and decreased after the infusion was completed. These patients may have benefited from a longer duration of infusion. Patients who died during the infusion were severely protein $\mathrm{C}$ deficient at baseline and 


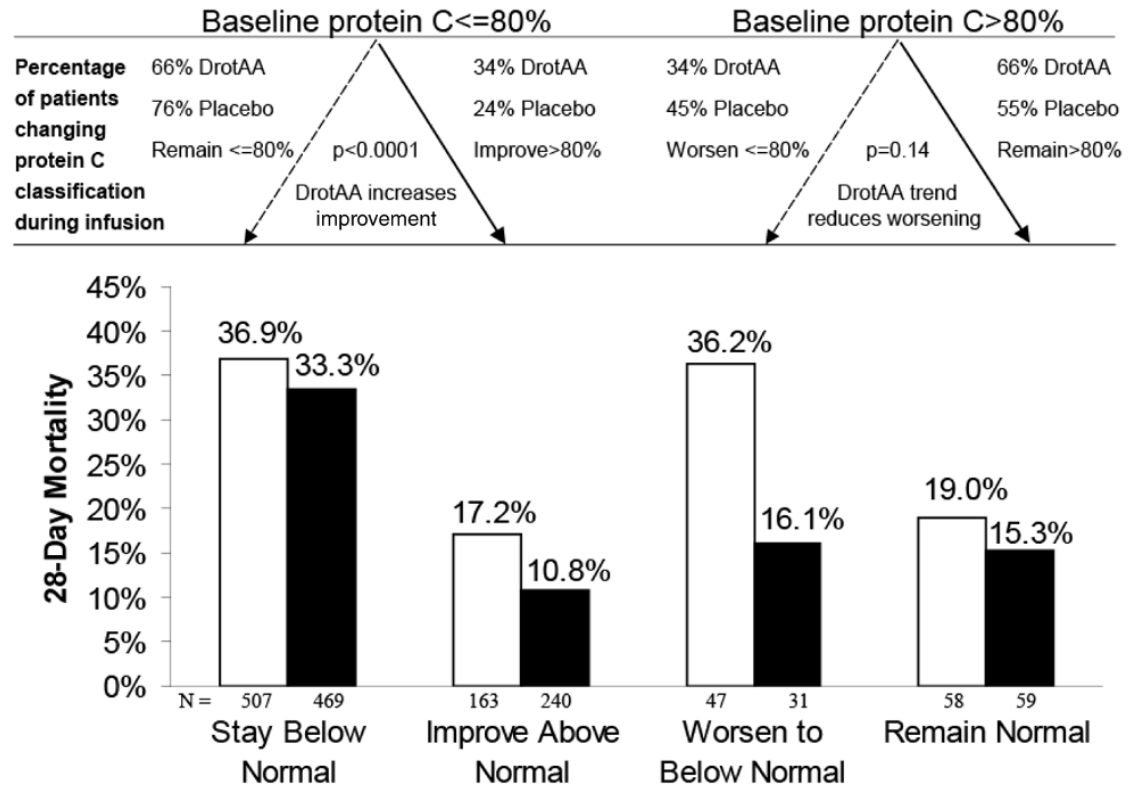

$\square$ Placebo DrotAA

Change in protein $\mathrm{C}$ levels and 28-day mortality in PROWESS patients [9]. DrotAA, drotrecogin alfa (activated); $\mathrm{N}$, total number of patients in each group; PROWESS, Recombinant Human Activated Protein C Worldwide Evaluation in Severe Sepsis.

Figure 3

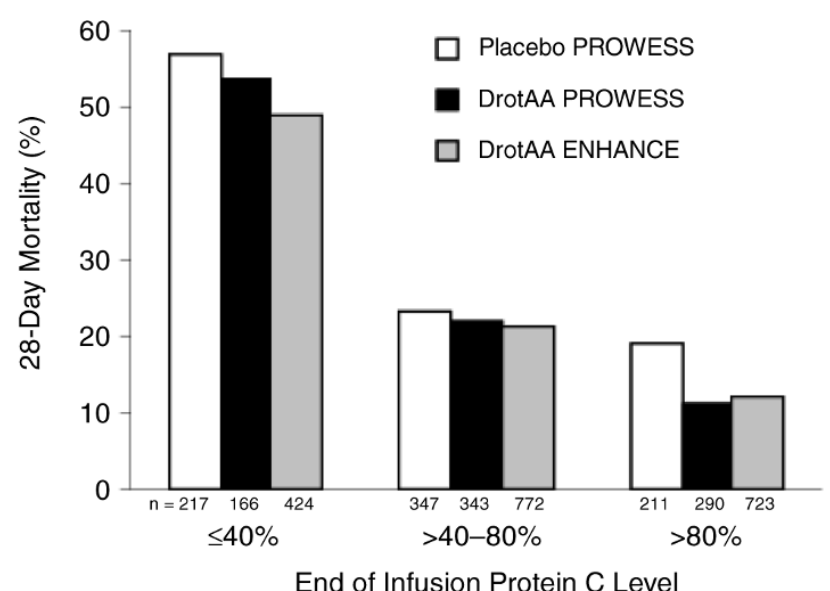

Mortality by end of infusion protein C level in PROWESS [9] and ENHANCE [10]. Patients were included if they had a baseline protein $\mathrm{C}$ measure. Day 4 protein $\mathrm{C}$ was classified as end of infusion. If the protein $\mathrm{C}$ level on day 4 was not available, then last observation carried forward values were used for classification. In this scale, $>80 \%$ is a normal protein $C$ level and $\leq 40 \%$ is half or less of the lower limit of normal. DrotAA, drotrecogin alfa (activated); ENHANCE, Extended Evaluation of Recombinant Human Activated Protein C, drotrecogin alfa (activated); N, total number of patients in each group; PROWESS, Recombinant Human Activated Protein C Worldwide Evaluation in Severe Sepsis.
Figure 4

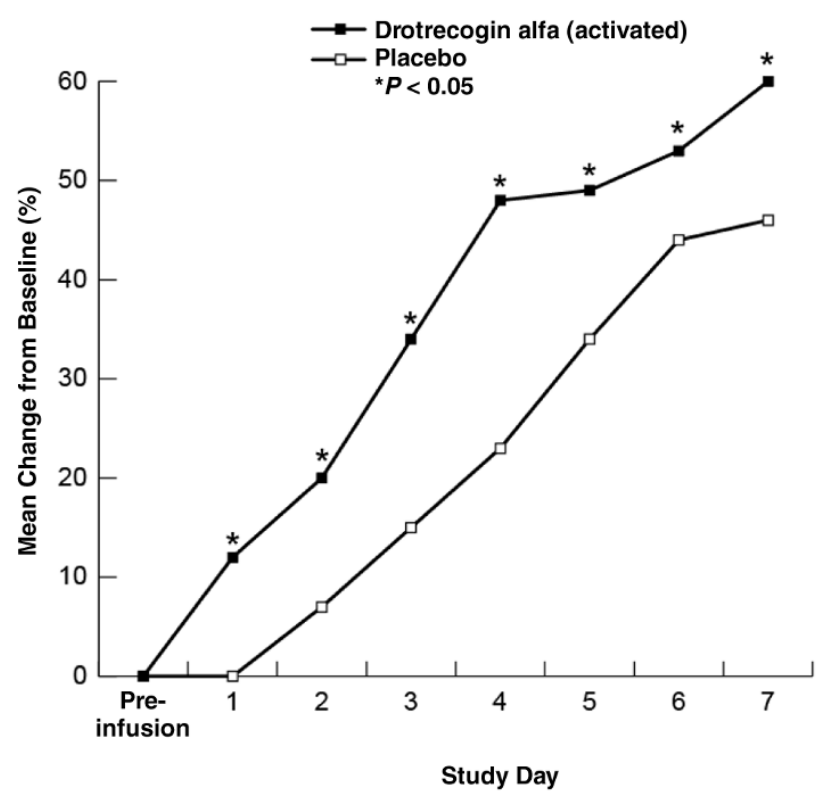

Effect of DrotAA infusion on protein $\mathrm{C}$ levels in PROWESS [9]. Shown are percentage changes in protein $\mathrm{C}$ level from baseline until day 7 ; the data are the observed data with no imputation. DrotAA, drotrecogin alfa (activated); PROWESS, Recombinant Human Activated Protein C Worldwide Evaluation in Severe Sepsis. 


\section{Figure 5}

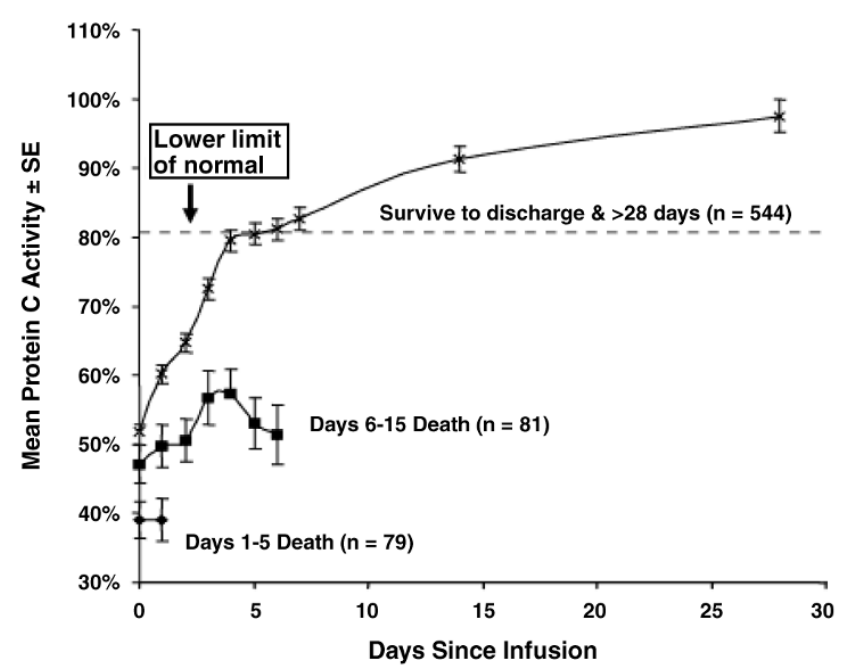

Mean protein $\mathrm{C}$ activity over time for selected PROWESS DrotAA patients classified by timing of death. Presented are means and standard errors (SE) of protein $C$ levels based on time of death of PROWESS [9] DrotAA patients. Raw values with no imputation are included. Note that not all groups are shown. DrotAA, drotrecogin alfa (activated); PROWESS, Recombinant Human Activated Protein C Worldwide Evaluation in Severe Sepsis.

exhibited no increase in protein $\mathrm{C}$ level during the infusion. These patients would not have had the opportunity to benefit from a longer infusion duration alone, but they may have benefited from an increased dose of DrotAA together, perhaps, with a longer infusion. Such hypotheses have been incorporated into the RESPOND study design.

\section{The RESPOND study}

RESPOND, a phase II double blind study aiming to recruit 488 patients, is the first step toward exploring the use of endogenous protein $\mathrm{C}$ level as a biomarker to guide the administration (both dosage and duration) of DrotAA. The study is organized into three periods: pretreatment, treatment and post-treatment. To be eligible for inclusion in the study, patients must have multiple organ dysfunction and protein $\mathrm{C}$ deficiency (protein $\mathrm{C}$ level less than the lower limit of normal). Protein $\mathrm{C}$ levels will be measured at each investigative site using a point-of-care immunoassay or a standard laboratory analyzer using an activity-based diagnostic kit.

The pretreatment period starts with the onset of multiple organ dysfunction (two or more sepsis-induced organ dysfunctions). After the onset of the second sepsis-induced organ dysfunction, a maximum of 24 hours is allowed to assess patient eligibility for inclusion in the study, obtain informed consent, perform baseline evaluations and determine patient treatment assignment. No more than 36 hours can elapse between the onset of the first sepsis-induced organ dysfunction and the start of the treatment period.
The treatment period begins with a common therapy lead-in, in which all patients will receive DrotAA at a dose of $24 \mu \mathrm{g} / \mathrm{kg}$ per hour for 24 hours. The rationale for this is underpinned by the observations from PROWESS that approximately onethird of patients who had severe protein $C$ deficiency at baseline were no longer severely deficient after 24 hours of DrotAA therapy, and that approximately one-quarter of patients who were not severely deficient at baseline were deficient at 24 hours, despite DrotAA treatment. This allows an initial assessment of response to standard dose therapy (24 $\mathrm{gg} / \mathrm{kg}$ per hour DrotAA).

After the 24-hour lead-in period, patients will receive the treatment that they were randomly assigned to (at baseline), either standard or alternative therapy. The alternative therapy received will depend on whether the patient's protein $\mathrm{C}$ level at the end of the lead-in period is less or greater than half the lower limit of normal, as demonstrated in Figure 1.

All patients assigned to standard therapy will receive an additional 72-hour infusion of DrotAA $24 \mu \mathrm{g} / \mathrm{kg}$ per hour (for a total infusion duration of 96 hours).

Patients assigned to receive alternative therapy of variable duration (the alternative therapy-variable duration arm), who have moderate protein $\mathrm{C}$ deficiency at the end of the lead-in period, will receive DrotAA $24 \mu \mathrm{g} / \mathrm{kg}$ per hour for an additional 24 to 144 hours. The treatment will be continued until two consecutive protein $\mathrm{C}$ levels are greater than or equal to the lower limit of normal, or the patient has received DrotAA for a total infusion duration of 168 hours. Based on results from PROWESS, this group of patients is anticipated to have a relatively low risk for early death (6.3\% at day 5 and $20.0 \%$ at day 28 , Figure 5 ), and therefore they will only be exposed to variable duration of DrotAA infusion and not a higher dose.

Patients assigned to alternative therapy at higher dose and of variable duration (the alternative therapy-higher dose/variable duration arm), who have severe protein $\mathrm{C}$ deficiency at the end of the lead-in period, will receive a variable duration of DrotAA infusion (as described above for the alternative therapy-variable duration arm) and a higher dose of DrotAA (30 $\mu \mathrm{g} / \mathrm{kg}$ per hour up to $48 \mu \mathrm{g} / \mathrm{kg}$ per hour). Based on results from PROWESS this group is anticipated to have a relatively high risk for early death (18.8\% at day 5 and $33.6 \%$ at day 28 , Figure 5), and therefore this group will be exposed to both higher doses and a variable duration of DrotAA infusion.

Each patient's infusion will be administered at a single infusion rate. A data monitoring committee will review safety data from the lower dose groups before enrolling patients in the next higher dose group. Figure 6 illustrates the different arms of the trial after completion of the common lead-in period, and shows the outcomes of corresponding PROWESS patients, indicating lower mortality among 


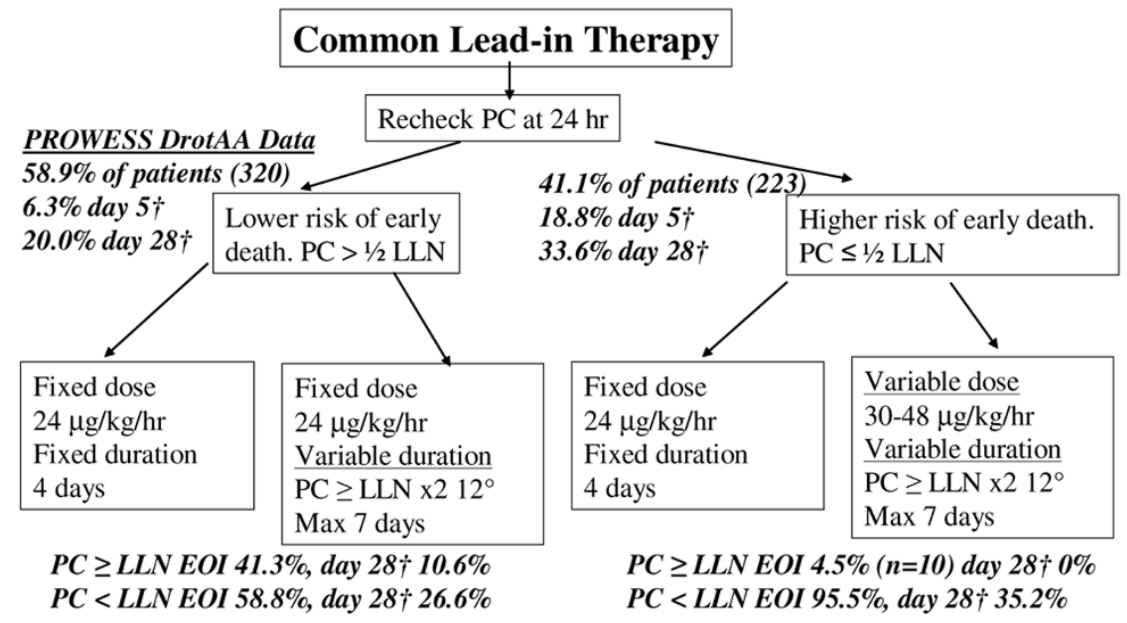

RESPOND study design. Distribution and outcome of corresponding DrotAA (Xigris ${ }^{\circledR}$ )-treated PROWESS [9] patients. DrotAA, drotrecogin alfa (activated); EOI, end of infusion; LLN, lower limit of normal; PC, protein C; PROWESS, Recombinant Human Activated Protein C Worldwide Evaluation in Severe Sepsis; RESPOND, Research Evaluating Serial Protein C levels in severe sepsis patients ON Drotrecogin alfa [activated]. †Mortality.

patients in whom protein $\mathrm{C}$ levels normalized by the end of the infusion period.

Shrouded infusion bags will be provided to the ICU by an unblinded pharmacist, who will make up a DrotAA or normal saline infusion depending on the most recent protein $C$ level and the stage of the study. This will ensure that the investigative staff will remain blinded to the study treatment.

RESPOND seeks to demonstrate that 'alternative therapy' results in a greater increase in protein $\mathrm{C}$ levels than 'standard therapy' and, importantly, to provide appropriate safety and efficacy data to determine the most appropriate aspects of alternative therapy to incorporate into possible future studies. If RESPOND is successful, then a larger phase 3 study will be required to demonstrate whether improved protein $C$ levels resulting from alternative therapy are also associated with statistically significant mortality reductions as compared with standard therapy. The ultimate aim of RESPOND and potential following studies is to establish serial plasma protein C measurements as a biomarker that will aid in the identification of those patients with severe sepsis who are most likely to benefit from DrotAA, that will enable adjustment of DrotAA therapy to the needs of individual patients (specifically, the possibility to use a higher dose and to adjust the infusion duration), and that will provide guidance to the clinician regarding whether the patient is responding to DrotAA therapy.

\section{Conclusion}

The future of medicine might be individualized therapy based on biomarkers or gene profiles. Until then, RESPOND represents the first step toward tailored sepsis treatment.

\section{Competing interests}

DW, AFS and KR have served as consultants and speakers for Eli Lilly and Company. DRN, JJ and BV are employed by Eli Lilly and Company.

\section{Acknowledgements}

This article is part of Critical Care Volume 11 Supplement 5: Severe sepsis and drotrecogin alfa (activated). The full contents of the supplement are available online at http://ccforum.com/supplements/11/S5. Publication of the supplement has been sponsored by Eli Lilly and Company.

\section{References}

1. Corrigan JJ, Ray WL, May N: Changes in the blood coagulation system associated with septicemia. N Engl J Med 1968, 279: 851-856.

2. Parrillo JE: Pathogenetic mechanisms of septic shock. $N$ Engl J Med 1993, 328:1471-1477.

3. Hinshaw LB: Sepsis/septic shock: participation of the microcirculation: an abbreviated review. Crit Care Med 1996, 24: 1072-1078.

4. Esmon CT: Inflammation and thrombosis: mutual regulation by protein C. Immunologist 1998, 6:84-89.

5. Esmon CT, Ding W, Yasuhiro K, Gu JM, Ferrell G, Regan LM, Stearns-Kurosawa DJ, Kurosawa S, Mather T, Laszik Z, Esmon NL: The protein C pathway: new insights. Thromb Haemost 1997, 78:70-74.

6. Macias WL, Yan SB, Williams MD, Um SL, Sandusky GE, Ballard DW, Planquois JMS: New insights into the protein $C$ pathway: potential implications for the biological activities of drotrecogin alfa (activated). Crit Care 2005, 9(suppl 4):S38-S45.

7. Mosnier LO, Zlokovic BV, Griffin JH: The cytoprotective protein C pathway. Blood 2007, 109:3161-3172.

8. Finigan $\mathrm{JH}$, Dudek SM, Singleton PA, Chiang ET, Jacobson JR, Camp SM, Ye SQ, Garcia JG: Activated protein C mediates novel lung endothelial barrier enhancement: role of sphingosine 1-phosphate receptor transactivation. J Biol Chem 2005, 280:17286-17293.

9. Bernard GR, Vincent JL, Laterre PF, LaRosa SP, Dhainaut JF, Lopez-Rodriguez A, Steingrub JS, Garber GE, Helterbrand JD, Ely EW, Fisher CJ Jr: Efficacy and safety of recombinant activated protein C for severe sepsis. N Engl J Med 2001, 344:699-709. 
10. Vincent JL, Bernard GR, Beale R, Doig C, Putensen C, Dhainaut JF, Artigas A, Fumagalli R, Macias W, Wright $T$, et al.: Drotrecogin alfa (activated) treatment in severe sepsis from the global open-label trial ENHANCE: further evidence for survival and safety and implications for early treatment. Crit Care Med 2005, 33:2266-2277.

11. Abraham E, Laterre PF, Garg R, Levy H, Talwar D, Trzaskoma BL, François B, Guy JS, Brückmann M, Rea-Neto A, et al.; for the Administration of Drotrecogin Alfa (Activated) in Early Stage Severe Sepsis (ADDRESS) Study Group: Drotrecogin alfa (activated) for adults with severe sepsis and a low risk of death. $N$ Engl J Med 2005, 353:1332-1341.

12. Nadel $S$, Goldstein $B$, Williams MD, Dalton $H$, Peters $M$, Macias WL, Abd-Allah SA, Levy H, Angle R, Wang D, et al.: Drotrecogin alfa (activated) in children with severe sepsis: a mulitcentre phase III randomized controlled trial. Lancet 2007, 369:836-843.

13. Levi M, Levy M, Williams MD, Douglas I, Artigas A, Antonelli M, Duncan W, Janes J, Booth FV, Wang D, et al:: Prophylactic heparin in patients with severe sepsis treated with drotrecogin alfa (activated). Am J Respir Crit Care Med 2007, 176:483-490.

14. Extended therapy of drotrecogin alfa (activated) 4 vs 7 days infusion (NCT00190788). [http://clinicaltrials.gov/ct2/show/ NCT00190788?intr=\%22Drotrecogin+alfa+activated\%22\&rank=3]

15. Evaluate impact to protein $\mathrm{C}$ levels with changing dose/duration of drotrecogin alfa (activated) administered to severe sepsis patients [http://clinicaltrials.gov/ct2/show/ NCT00386425? intr=\%22Protein+C\%22]

16. Sheth $\mathrm{SB}$, Carvalho $\mathrm{AC}$ : Protein $\mathrm{S}$ and $\mathrm{C}$ alterations in acutely ill patients. Am J Hematol 1991, 36:14-19.

17. Yan SB, Helterbrand JD, Hartman DL, Wright TJ, Bernard GD: Low levels of protein $C$ are associated with poor outcome in severe sepsis. Chest 2001, 120:915-922.

18. Kinasewitz GT, Yan SB, Basson B, Comp P, Russell JA, Cariou A Um SL, Utterback B, Laterre P-F, Dhainaut J-F; for the PROWESS Sepsis Study Group: Universal changes in biomarkers of coagulation and inflammation occur in patients with severe sepsis, regardless of causative micro-organism. Crit Care 2004, 8:R82-R90.

19. Fourrier F, Chopin C, Goudemand J, Hendrycx S, Caron C, Rime A, Marey A, Lestavel P: Septic shock, multiple organ failure, and disseminated intravascular coagulation: compared patterns of antithrombin III, protein $\mathbf{C}$ and protein $\mathbf{S}$ deficiencies. Chest 1992, 101:816-823.

20. Lorente JA, Garcia-Frade LJ, Landin L, de Pablo R, Torrado C, Renez E, Garcia-Avello A: Time course of hemostatic abnormalities in sepsis and its relation to outcome. Chest 1993, 103:1536-1542.

21. Mesters RM, Helterbrand J, Utterback BG, Yan SB, Chao YB, Fernandez JA, Griffin JH, Hartman DL: Prognostic value of protein C concentrations in neutropenic patients at high risk of severe septic complications. Crit Care Med 2000, 28:22092216.

22. Brandtzaeg $P$, Sandset PM, Joo GB, Ovstebo R, Abildgaard U, Kieruef $P$ : The quantitative association of plasma endotoxin, antithrombin, protein $\mathrm{C}$, extrinsic pathway inhibitor, and fibrinopeptide $A$ in systemic meningococcal disease. Thromb Res 1989, 55:459-470.

23. Philippe J, Offner F, Leroux-Roels G, Vogelaers D, Baele G: Plasminogen activator inhibitor-1, protein $C$, antithrombin III and tissue plasminogen activator activities in the early phase of septic shock [abstract]. Thromb Haemost 1989, 65:A1836.

24. Hesselvik JF, Malm J, Dahlback B, Blomback M: Protein C, protein $S$ and $C 4 b$-binding protein in severe infection and septic shock. Thromb Haemost 1991, 65:126-129.

25. Leclerc F, Hazelzet J, Jude B, Hofhuis W, Hue V, Martinot A, Van der Voort $E$ : Protein $\mathbf{C}$ and $\mathbf{S}$ deficiency in severe infectious purpura of children: a collaborative study of $\mathbf{4 0}$ cases. Intensive Care Med 1992, 18:202-205.

26. Roman J, Velasco F, Fernandez F, Fernandez M, Villalba R, Rubin $\mathrm{V}$, Torres $\mathrm{A}$ : Protein $\mathrm{C}$, protein $\mathrm{S}$, and $\mathrm{C} 4 \mathrm{~b}$-binding protein in neonatal severe infection and septic shock. J Perinat Med 1992, 20:111-116.

27. Fijnvandraat $K$, Derkx B, Peters M, Sturk A, Prins $M H$, van Deventer SJ, ten Cate JW: Coagulation activation and tissue necrosis in meningoccocal septic shock: severely reduced protein C levels predict a high mortality. Thromb Haemost 1995, 73:1520.
28. Piette WW, Shasby DM, Kealey GP, Olson JD: Retiform purpura is a sign of severe acquired protein $C$ deficiency and risk of progression to purpura fulminans in sepsis and disseminated intravascular coagulation [abstract]. Clin Res 1993, 41:253A.

29. Powars D, Larsen R, Johnson J, Hulbert T, Sun $T$, Patch $M$, Francis R, Chan L: Epidemic meningococcemia and purpura fulminans with induced protein C deficiency. Clin Infect Dis 1993, 17:254-261.

30. Hazelzet JA, Van der Voort E, Lindemans J, Ter Heerdt PGJ, Neijens HJ: Relation between cytokines and routine laboratory data in children with septic shock and purpura. Intensive Care Med 1994, 20:371-374.

31. Fijnvandraat K, Derkx B, Peters M, Bijlmer R, Sturk A, Prins MH, van Deventer SJ, ten Cate JW: Coagulation activation and tissue necrosis in meningococcal septic shock: severely reduced protein $\mathrm{C}$ levels predict a high mortality. Thromb Haemost 1995, 73:15-20.

32. Brunkhorst F, Sakr Y, Hagel S, Reinhart K: Protein C concentrations correlate with organ dysfunction and predict outcome independent of the presence of sepsis. Anesthesiology 2007, 107:15-23.

33. Ware LB, Matthay MA, Parsons PE, Thompson BT, Januzzi JL, Eisner MD; for the National Heart, Lung, and Blood Institute Acute Respiratory Distress Syndrome Clinical Trials Network: Pathogenetic and prognostic significance of altered coagulation and fibrinolysis in acute lung injury acute respiratory distress syndrome. Crit Care Med 2007, 35:1822-1828.

34. Macias WL, Nelson DR: Severe protein $C$ deficiency predicts early death in severe sepsis. Crit Care Med 2004, 32(suppl): S223-S228. 